\title{
Violation of the Kubo-Martin-Schwinger condition along a Rindler trajectory in polymer quantization
}

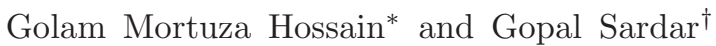 \\ Department of Physical Sciences, Indian Institute of Science Education and Research Kolkata, Mohanpur - 741 246, WB, India
}

(Dated: June 7, 2022)

\begin{abstract}
Existence of Unruh effect is often understood from the property of two-point function along Rindler trajectory where it satisfies KMS condition. In particular, it exhibits the so-called KMS periodicity along imaginary time direction. Corresponding period is then identified with reciprocal of Unruh temperature times Boltzmann constant. We show here that the two-point function including leading order perturbative corrections due to polymer quantization, the quantization method used in loop quantum gravity, violates KMS condition in low-energy regime. This violation is caused by correction terms which are not Lorentz invariants. Consequently, polymer corrected two-point function along Rindler trajectory looses its thermal interpretation. We discuss its implications on existence of Unruh effect in the context of polymer quantization.
\end{abstract}

PACS numbers: 04.62.+v, 04.60.Pp

\section{INTRODUCTION}

With respect to a uniformly accelerating observer, Fock vacuum state appears as a thermal state rather than a zero-particle state. This phenomena is referred as Unruh effect [1-6] and it is an important result of standard quantum field theory when applied to a curved spacetime [7, 8]. Unruh effect can be realized in many different ways. Firstly, one can employ the method of Bogoliubov transformation in which one computes expectation value of number density operator associated with the accelerating observer, in Fock vacuum state. The result of this computation turns out to be a blackbody distribution of a given temperature known as Unruh temperature. In this method, one needs to include contributions from transPlanckian modes as seen by an inertial observer. This particular aspect makes Unruh effect to be a potentially interesting arena for understanding and exploring implications of Planck-scale physics [9 11]. In particular, one may use it for probing a candidate theory of quantum gravity as trans-Planckian modes are expected to receive significant modifications from it. While the method of Bogoliubov transformation is conceptually simpler but it requires sophisticated regularization techniques to deal with associated field theoretical divergences. Therefore, it is often imperative to understand the origin of Unruh effect using different methods.

In quantum statistical mechanics, it is well known that Gibbs ensemble average of two-point function which is in thermal equilibrium with a reservoir satisfies the socalled Kubo-Martin-Schwinger (KMS) condition [12 14]. In particular, thermal two-point function exhibits periodicity with a twist along imaginary time direction 15]. Corresponding period is then identified with reciprocal of reservoir temperature times Boltzmann constant. Subse-

\footnotetext{
*Electronic address: ghossain@iiserkol.ac.in
}

${ }^{\dagger}$ Electronic address: gopal1109@iiserkol.ac.in quently, this property is used in reverse to argue that if a two-point function computed in a state, satisfies KMS condition then one can associate a thermal characteristic to the state. As an example, it can be shown that two-point function computed in Fock vacuum state and when viewed along the trajectory of a uniformly accelerating observer, appears like a thermal two-point function [16, 17] as it satisfies KMS condition. The corresponding KMS period then leads to a reservoir temperature which is precisely equal to Unruh temperature.

As mentioned earlier, in the method of Bogoliubov transformation, one needs to include trans-Planckian modes. These modes are expected to be modified significantly by Planck-scale physics. Therefore, one is naturally led to ask whether the effects from a theory of Planck scale physics can alter a few specific properties of two-point function even in low-energy regime. In particular, one can ask whether the corrections from such a theory can lead to a violation of KMS condition. In this paper, we perform the task in the context of polymer quantization.

Polymer quantization or loop quantization [18, 19] is a quantization method which is used in loop quantum gravity [20 22]. It differs from Schrödinger quantization in several aspects when applied to a mechanical system. In particular, apart from Planck constant $\hbar$, it comes with a new dimension-full parameter. In the context of full quantum gravity, this new scale would correspond to Planck length. Secondly, kinematical Hilbert space being non-separable, one cannot define both position and momentum operators simultaneously in polymer quantization but only one of them. Due to the non-separability of kinematical Hilbert space, the Stone-von Neumann uniqueness theorem is also not applicable. These aspects make polymer quantization unitarily inequivalent to Schrödinger quantization [18]. Therefore, in principle, one can get a different set of results from polymer quantization.

In usual derivation of two-point function in Fock space, the field operator is expressed in terms of creation and 
annihilation operators of different Fourier modes. Each of these modes behaves as a mechanical system corresponding to a decoupled harmonic oscillator. Subsequently these modes are quantized using Schrödinger quantization method. In polymer quantization of these modes, the notions of creation and annihilation operators are not readily available. Therefore, following reference [23], we use a slightly improvised method in which twopoint function is derived using energy spectrum of these modes.

In section II we briefly review some aspects of a uniformly accelerating observer and its associated Rindler spacetime. In section III, we revisit KMS condition in the context of quantum statistical mechanics. In section [IV] we consider a massless scalar field to compute twopoint function in both Fock and polymer quantization. Then we show that polymer corrected two-point function along Rindler trajectory does not satisfy KMS condition. Consequently, polymer corrected two-point function for Rindler observer looses its thermal interpretation. The results shown here is consistent with the result of 24] where it is shown using method of Bogoliubov transformation that Unruh effect is absent in polymer quantization.

\section{RINDLER OBSERVER}

A section of Minkowski spacetime when viewed from a uniformly accelerating frame, can be described by Rindler metric. Using conformal Rindler coordinates $\bar{x}^{\alpha}=(\tau, \xi, y, z) \equiv(\tau, \vec{\xi})$ together with natural units $(c=\hbar=1)$, Rindler metric can be expressed as [25]

$$
d s^{2}=e^{2 a \xi}\left(-d \tau^{2}+d \xi^{2}\right)+d y^{2}+d z^{2} \equiv g_{\alpha \beta} d \bar{x}^{\alpha} d \bar{x}^{\beta} .
$$

The parameter $a$ here denotes magnitude of acceleration 4 -vector. To an inertial observer who uses the coordinates $x^{\mu}=(t, x, y, z) \equiv(t, \mathbf{x})$, the Minkowski metric would appear as $d s^{2}=\eta_{\mu \nu} d x^{\mu} d x^{\nu}=-d t^{2}+d x^{2}+d y^{2}+$ $d z^{2}$. If the uniformly accelerating observer, referred as Rindler observer, moves along +ve $\mathrm{x}$-axis with respect to the inertial observer then their coordinates are related to each other as

$$
t=\frac{1}{a} e^{a \xi} \sinh a \tau, \quad x=\frac{1}{a} e^{a \xi} \cosh a \tau .
$$

The $y$ and $z$ coordinates are related trivially. The equation (2) makes it clear that Rindler spacetime covers only a wedge-shaped region, referred as Rindler wedge, of Minkowski spacetime.

\section{KMS CONDITION}

In quantum statistical mechanics, Gibbs ensemble average of an observable $\hat{O}$ which is in thermal equilibrium with a reservoir of temperature $T$, can be expressed as

$$
\langle\hat{O}\rangle_{\beta}=Z^{-1} \operatorname{Tr}\left[e^{-\beta \hat{H}} \hat{O}\right]
$$

where $\beta=1 / k_{B} T, \hat{H}$ is the associated Hamiltonian operator and $Z=\operatorname{Tr}\left[e^{-\beta \hat{H}}\right]$ is the corresponding partition function. Here $k_{B}$ refers to Boltzmann constant. If we consider the observable to be $\hat{O}=\hat{\phi}(\tau, \vec{\xi}) \hat{\phi}\left(\tau^{\prime}, \overrightarrow{\xi^{\prime}}\right)$ where $\hat{\phi}(\tau, \vec{\xi})$ is a given field operator, then thermal two-point function associated with the field can be expressed as

$$
\left\langle\hat{\phi}(\tau, \vec{\xi}) \hat{\phi}\left(\tau^{\prime}, \vec{\xi}^{\prime}\right)\right\rangle_{\beta}=Z^{-1} \operatorname{Tr}\left[e^{-\beta \hat{H}} \hat{\phi}(\tau, \vec{\xi}) \hat{\phi}\left(\tau^{\prime}, \vec{\xi}^{\prime}\right)\right]
$$

Using time evolution of the field operator as $\hat{\phi}(\tau, \vec{\xi})=$ $e^{i \hat{H} \tau} \hat{\phi}(0, \vec{\xi}) e^{-i \hat{H} \tau}$ and invariance of trace under cyclic permutation, one can easily show that

$$
\left\langle\hat{\phi}(\tau, \vec{\xi}) \hat{\phi}\left(\tau^{\prime}, \vec{\xi}^{\prime}\right)\right\rangle_{\beta}=\left\langle\hat{\phi}\left(\tau^{\prime}, \vec{\xi}^{\prime}\right) \hat{\phi}(\tau+i \beta, \vec{\xi})\right\rangle_{\beta} .
$$

The equation (5) is commonly referred as Kubo-MartinSchwinger (KMS) condition [13, 14]. One may note that relative position of field operators in two sides are interchanged. Therefore, corresponding periodicity of twopoint function along imaginary time direction (5) is often referred as periodicity with a twist [15].

\section{MASSLESS SCALAR FIELD}

The dynamics of a massless scalar field $\Phi(x)$ in Minkowski spacetime is governed by the action

$$
S_{\Phi}=\int d^{4} x\left[-\frac{1}{2} \sqrt{-\eta} \eta^{\mu \nu} \partial_{\mu} \Phi(x) \partial_{\nu} \Phi(x)\right] .
$$

In this paper, we are mainly interested in employing polymer quantization scheme which is a canonical quantization method. Therefore, it is necessary for us to compute the Hamiltonian associated with the action (6). By considering spatial hyper-surfaces which are labeled by $t$, one can compute corresponding Hamiltonian as

$$
H_{\Phi}=\int d^{3} \mathbf{x}\left[\frac{\Pi^{2}}{2 \sqrt{q}}+\frac{\sqrt{q}}{2} q^{a b} \partial_{a} \Phi \partial_{b} \Phi\right]
$$

where $q_{a b}$ is the metric on spatial hyper-surfaces. Poisson bracket between the field $\Phi=\Phi(t, \mathbf{x})$ and the conjugate field momentum $\Pi=\Pi(t, \mathbf{x})$ are given by

$$
\{\Phi(t, \mathbf{x}), \Pi(t, \mathbf{y})\}=\delta^{3}(\mathbf{x}-\mathbf{y})
$$

\section{A. Fourier modes}

We define Fourier modes for the scalar field and its conjugate momentum in Minkowski spacetime as

$$
\Phi=\frac{1}{\sqrt{V}} \sum_{\mathrm{k}} \tilde{\phi}_{\mathrm{k}}(t) e^{i \mathrm{k} \cdot \mathbf{x}}, \Pi=\frac{1}{\sqrt{V}} \sum_{\mathrm{k}} \sqrt{q} \tilde{\pi}_{\mathrm{k}}(t) e^{i \mathrm{k} \cdot \mathbf{x}},
$$


where $V=\int d^{3} \mathbf{x} \sqrt{q}$ is the spatial volume. For Minkowski spacetime, the spatial volume diverges given the space is non-compact. Therefore, in order to avoid dealing with divergent quantity, one considers a fiducial box of finite volume. In such situation, Kronecker delta and Dirac delta are expressed as $\int d^{3} \mathbf{x} \sqrt{q} e^{i\left(\mathrm{k}-\mathrm{k}^{\prime}\right) \cdot \mathbf{x}}=$ $V \delta_{\mathrm{k}, \mathrm{k}^{\prime}}$ and $\sum_{\mathrm{k}} e^{i \mathrm{k} \cdot(\mathbf{x}-\mathbf{y})}=V \delta^{3}(\mathbf{x}-\mathbf{y}) / \sqrt{q}$. In terms of Fourier modes (9), the Hamiltonian (7) can be expressed as $H_{\Phi}=\sum_{\mathrm{k}} \mathcal{H}_{\mathrm{k}}$, where Hamiltonian density for the $\mathrm{k}-$ th mode is

$$
\mathcal{H}_{\mathrm{k}}=\frac{1}{2} \tilde{\pi}_{-\mathrm{k}} \tilde{\pi}_{\mathrm{k}}+\frac{1}{2}|\mathrm{k}|^{2} \tilde{\phi}_{-\mathrm{k}} \tilde{\phi}_{\mathrm{k}}
$$

Corresponding Poisson bracket is given by

$$
\left\{\tilde{\phi}_{\mathrm{k}}, \tilde{\pi}_{-\mathrm{k}^{\prime}}\right\}=\delta_{\mathrm{k}, \mathrm{k}^{\prime}} \text {. }
$$

By redefining complex-valued modes $\tilde{\phi}_{\mathrm{k}}$ and momenta $\tilde{\pi}_{\mathrm{k}}$ in terms of real-valued functions $\phi_{\mathrm{k}}$ and $\pi_{\mathrm{k}}$ such that reality condition of the scalar field $\Phi$ is ensured, one can express Hamiltonian density and Poisson bracket as

$$
\mathcal{H}_{\mathrm{k}}=\frac{1}{2} \pi_{\mathrm{k}}^{2}+\frac{1}{2}|\mathrm{k}|^{2} \phi_{\mathrm{k}}^{2} \quad ; \quad\left\{\phi_{\mathrm{k}}, \pi_{\mathrm{k}^{\prime}}\right\}=\delta_{\mathrm{k}, \mathrm{k}^{\prime}} .
$$

This is the usual Hamiltonian for a set of decoupled harmonic oscillators. If we denote energy spectrum of these quantum oscillators as $\hat{\mathcal{H}}_{\mathrm{k}}\left|n_{\mathrm{k}}\right\rangle=E_{n}^{(\mathrm{k})}\left|n_{\mathrm{k}}\right\rangle$ then corresponding vacuum state can be expressed as $|0\rangle=$ $\Pi_{\mathrm{k}} \otimes\left|0_{\mathrm{k}}\right\rangle$.

\section{B. Two-point function in Minkowski spacetime}

In position space two-point function using Minkowski coordinates can be written as

$$
G\left(x, x^{\prime}\right) \equiv\left\langle 0\left|\hat{\Phi}(x) \hat{\Phi}\left(x^{\prime}\right)\right| 0\right\rangle=\left\langle 0\left|\hat{\Phi}(t, \mathbf{x}) \hat{\Phi}\left(t^{\prime}, \mathbf{x}^{\prime}\right)\right| 0\right\rangle
$$

where $|0\rangle$ denotes corresponding vacuum state. Using definition of Fourier modes, one can express two-point function (13) as

$$
G\left(x, x^{\prime}\right)=\frac{1}{V} \sum_{\mathrm{k}} D_{\mathrm{k}}\left(t, t^{\prime}\right) e^{i \mathrm{k} \cdot\left(\mathbf{x}-\mathbf{x}^{\prime}\right)},
$$

where the matrix element can be written as

$$
D_{\mathrm{k}}\left(t, t^{\prime}\right)=\left\langle 0_{\mathrm{k}}\left|e^{i \hat{\mathcal{H}}_{\mathrm{k}} t} \hat{\phi}_{\mathrm{k}} e^{-i \hat{\mathcal{H}}_{\mathrm{k}} t} e^{i \hat{\mathcal{H}}_{\mathrm{k}} t^{\prime}} \hat{\phi}_{\mathrm{k}} e^{-i \hat{\mathcal{H}}_{\mathrm{k}} t^{\prime}}\right| 0_{\mathrm{k}}\right\rangle .
$$

We now remove the fiducial volume by taking the limit $V \rightarrow \infty$. This essentially requires that we replace the sum $\frac{1}{V} \sum_{\mathrm{k}}$ by an integration $\int \frac{d^{3} \mathrm{k}}{(2 \pi)^{3}}$. Thanks to the definition of Fourier modes (9), the equation (12) is independent of the fiducial volume. Therefore, two-point function (14) becomes

$$
G\left(x, x^{\prime}\right)=\int \frac{d^{3} \mathrm{k}}{(2 \pi)^{3}} D_{\mathrm{k}}\left(t, t^{\prime}\right) e^{i \mathrm{k} \cdot\left(\mathbf{x}-\mathbf{x}^{\prime}\right)} .
$$

Using energy spectrum and expanding the state $\hat{\phi}_{\mathrm{k}}\left|0_{\mathrm{k}}\right\rangle$ in the basis of energy eigenstates as $\hat{\phi}_{\mathrm{k}}\left|0_{\mathrm{k}}\right\rangle=\sum_{n} c_{n}\left|n_{\mathrm{k}}\right\rangle$, one can reduce the matrix element as

$$
D_{\mathrm{k}}\left(t-t^{\prime}\right) \equiv D_{\mathrm{k}}\left(t, t^{\prime}\right)=\sum_{n}\left|c_{n}\right|^{2} e^{-i \Delta E_{n}\left(t-t^{\prime}\right)},
$$

where $\Delta E_{n} \equiv E_{n}^{(\mathrm{k})}-E_{0}^{(\mathrm{k})}$ and $c_{n}=\left\langle n_{\mathrm{k}}\left|\hat{\phi}_{\mathrm{k}}\right| 0_{\mathrm{k}}\right\rangle$. We shall see later that $\mathrm{k}$ dependence of $D_{\mathrm{k}}\left(t-t^{\prime}\right)$ is through $|\mathrm{k}|$. So above integration can be evaluated conveniently by using polar coordinates in momentum space as

$$
G\left(x, x^{\prime}\right)=\int \frac{k^{2} d k}{4 \pi^{2}} D_{k}(\Delta t) \int \sin \theta d \theta e^{i k|\Delta \mathbf{x}| \cos \theta},
$$

where $k=|\mathrm{k}|, \Delta \mathbf{x}=\mathbf{x}-\mathbf{x}^{\prime}$ and $\Delta t=t-t^{\prime}$. By carrying out $\theta$ integration one can express the two-point function as

$$
G\left(x, x^{\prime}\right)=G_{+}-G_{-},
$$

where

$$
G_{ \pm}=\frac{i}{4 \pi^{2}|\Delta \mathbf{x}|} \int d k k D_{k}(\Delta t) e^{\mp i k|\Delta \mathbf{x}|}
$$

\section{Fock quantization}

In Fock space, Fourier modes, as represented by equation (12), are quantized using Schrödinger quantization method. It is straightforward to compute corresponding energy spectrum and the coefficients $c_{n}$ as

$$
E_{\mathrm{k}}^{n}=\left(n+\frac{1}{2}\right)|\mathrm{k}| ; \Delta E_{n}=n|\mathrm{k}| ; \quad c_{n}=\frac{\delta_{1, n}}{\sqrt{2|\mathrm{k}|}}
$$

The expressions as given in (21), led to a simpler form of the matrix element

$$
D_{\mathrm{k}}(\Delta t)=\frac{1}{2|\mathrm{k}|} e^{-i|\mathrm{k}| \Delta t}
$$

Along a timelike trajectory $(\Delta t \pm|\Delta \mathbf{x}|)$ is always positive when $\Delta t>0$. So by defining a new variable $u=k(\Delta t \pm$ $|\Delta \mathbf{x}|)$, we can transform the expression of $G_{ \pm}$(20) in Fock space as

$$
G_{ \pm}=\frac{i}{8 \pi^{2}|\Delta \mathbf{x}|} \frac{I_{0}}{(\Delta t \pm|\Delta \mathbf{x}|)}
$$

where $I_{n}=\int_{0}^{\infty} d u u^{n} e^{-i u}$. We need to evaluate $I_{n}$ only for $n=0$ here. However for later convenience, we evaluate $I_{n}$ for $n \geq 0$. Firstly we note that integral expression of $I_{n}$ is formally divergent. Therefore, in order to regulate it, we introduce a small, positive parameter $\epsilon$ in the integrand such that regulated expression of $I_{n}$ becomes

$$
I_{n}^{\epsilon}=\int_{0}^{\infty} d u u^{n} e^{-i u-\epsilon u}=\frac{\Gamma(n+1)}{(i+\epsilon)^{n+1}} .
$$


Using regulated $I_{n}^{\epsilon}$, it is straightforward to simplify the two-point function as

$$
G\left(x, x^{\prime}\right)=\frac{(1-i \epsilon)^{-1}}{4 \pi^{2} \Delta x^{2}},
$$

where $\Delta x^{2}=-\Delta t^{2}+|\Delta \mathbf{x}|^{2}$ is Lorentz invariant spacetime interval.

\section{Polymer quantization}

Polymer quantization comes with a new dimension-full parameter say $l_{\star}$ which is analogous of Planck length in full quantum gravity. For each Fourier mode, one may define a dimensionless parameter $g=|\mathrm{k}| l_{\star}$. It signifies the scale of polymer corrections. Energy eigenvalues for $\mathrm{k}$-th oscillator in polymer quantization [23] are given by

$$
\frac{E_{\mathrm{k}}^{2 n}}{|\mathrm{k}|}=\frac{1}{4 g}+\frac{g}{2} A_{n}(g), \frac{E_{\mathrm{k}}^{2 n+1}}{|\mathrm{k}|}=\frac{1}{4 g}+\frac{g}{2} B_{n+1}(g),
$$

where $n \geq 0, A_{n}$ and $B_{n}$ are Mathieu characteristic value functions. The corresponding energy eigenfunctions are $\psi_{2 n}(v)=\operatorname{ce}_{n}\left(1 / 4 g^{2}, v\right) / \sqrt{\pi}$ and $\psi_{2 n+1}(v)=$ $\operatorname{se}_{n+1}\left(1 / 4 g^{2}, v\right) / \sqrt{\pi}$ where $v=\pi_{\mathrm{k}} \sqrt{l_{\star}}+\pi / 2$. The functions ce ce $_{n}$ and $\mathrm{se}_{n}$ are solutions to Mathieu equation. They are referred as elliptic cosine and sine functions respectively [26]. We may emphasize here that in order to arrive at these $\pi$-periodic and $\pi$-antiperiodic states in $v$, one invokes superselection rules motivated by the symmetry of associated potential in energy eigenvalue equation. In particular, the potential when expressed in terms of the variable $v$, is periodic with period $\pi$. The corresponding energy eigenvalues are then expressed exactly in terms of Mathieu characteristic value functions $A_{n}$ and $B_{n}$. Having exact energy spectrum allows one to study different aspects of the system analytically. Furthermore, without imposition of superselection rules some aspects of the given system are known to be problematic [27]. In particular, it is shown that even basic notions of statistical entropy, canonical partition function for such a system become ill-defined due to the effective infinite degeneracy of the energy eigenvalues in polymer quantization, unless one invokes some superselection rules [27].

Unlike Fock space, matrix element $D_{\mathrm{k}}(\Delta t)$ (17) does not appear to be exactly summable given all $c_{4 n+3}=$ $i \sqrt{l_{\star}} \int_{0}^{2 \pi} \psi_{4 n+3} \partial_{v} \psi_{0} d v$ (for $n=0,1,2, \ldots$ ) are nonvanishing in polymer quantization. However, asymptotic properties of Mathieu functions are well known and they may be used to evaluate the matrix element approximately. Following reference [23], we note that for lowenergy modes (compared to $l_{\star}$, i.e. $g \ll 1$ )

$$
\frac{\Delta E_{4 n+3}}{|\mathrm{k}|}=(2 n+1)-\frac{(4 n+3)^{2}-1}{16} g+\mathcal{O}\left(g^{2}\right),
$$

for $n \geq 0$, and

$$
c_{3}=\frac{i}{\sqrt{2|\mathrm{k}|}}[1+\mathcal{O}(g)], \frac{c_{4 n+3}}{c_{3}}=\mathcal{O}\left(g^{n}\right),
$$

for $n>0$. On the other hand for trans-Planckian modes (i.e. $g \gg 1$ ), we note that

$$
\frac{\Delta E_{4 n+3}}{|\mathrm{k}|}=2(n+1)^{2} g+\mathcal{O}\left(\frac{1}{g^{3}}\right),
$$

for $n \geq 0$, and

$$
c_{3}=i \sqrt{\frac{g}{2|\mathrm{k}|}}\left[\frac{1}{4 g^{2}}+\mathcal{O}\left(\frac{1}{g^{6}}\right)\right], \frac{c_{4 n+3}}{c_{3}}=\mathcal{O}\left(\frac{1}{g^{2 n}}\right),
$$

for $n>0$. So in both asymptotic cases, leading contributions to the expression of $D_{\mathrm{k}}(\Delta t)$ (17) comes from $c_{3}$ term compared to other non-vanishing $c_{4 n+3}$ terms. Therefore, one can approximate matrix element $D_{\mathrm{k}}(\Delta t)$ in polymer quantization as

$$
D_{\mathrm{k}}^{\text {poly }}(\Delta t) \simeq\left|c_{3}\right|^{2} e^{-i \Delta E_{3} \Delta t} .
$$

Further, we note that for low-energy modes $(g \ll 1)$, modulus of the integrand in equation (20) varies as

$$
\left|k D_{\mathrm{k}}^{\text {poly }}(\Delta t)\right|=\frac{1}{2}[1+\mathcal{O}(g)],
$$

whereas for trans-Planckian modes $(g \gg 1)$, modulus of the integrand varies as

$$
\left|k D_{\mathrm{k}}(\Delta t)\right|=\frac{1}{32 g^{3}}\left[1+\mathcal{O}\left(\frac{1}{g^{4}}\right)\right] \ll 1 .
$$

So to evaluate the expression of $G_{ \pm}(20)$ in polymer quantization, one may restrict the integration along $k$ up to a pivotal $k_{\max }$. In other words, polymer corrected superPlanckian modes do not contribute significantly towards two-point function as expected. Nevertheless, each lowenergy mode that does contribute to the two-point function, comes with perturbative polymer corrections.

In order to understand the implications of each correction terms separately, we parameterize the perturbative polymer corrections to energy spectrum and coefficient $c_{3}$ as

$$
\Delta E_{3}^{\star}=k\left(1+\delta_{E 3} l_{\star} k\right) ;\left|c_{3}\right|_{\star}^{2}=\frac{1}{2 k}\left(1+2 \delta_{c 3} l_{\star} k\right),
$$

where $\delta_{E 3}$ and $\delta_{c 3}$ are numerical constants signifying the deviation from Fock quantization and they carry the label of their origin. We now express $G_{ \pm}(20)$ with perturbative polymer corrections as

$$
G_{ \pm}^{p o l y} \simeq \frac{i}{4 \pi^{2}|\Delta \mathbf{x}|} \int_{0}^{k_{\max }} d k k D_{k}^{\star}(\Delta t) e^{\mp i k|\Delta \mathbf{x}|}
$$

where $D_{\mathrm{k}}^{\star}(\Delta t)=\left|c_{3}\right|_{\star}^{2} e^{-i \Delta E_{3}^{\star} \Delta t}$. By defining a new variable $u=k\left(\Delta t \pm|\Delta \mathbf{x}|+\delta_{E 3} l_{\star} k \Delta t\right)$, one can transform the integral (35) as

$$
\begin{aligned}
& G_{ \pm}^{\text {poly }}\left(x, x^{\prime}\right) \simeq \frac{i}{8 \pi^{2}|\Delta \mathbf{x}|}\left[\frac{I_{0}\left(u_{0}^{ \pm}\right)}{(\Delta t \pm|\Delta \mathbf{x}|)}+\right. \\
& \left.2 l_{\star} I_{1}\left(u_{0}^{ \pm}\right)\left\{\frac{\delta_{c 3}}{(\Delta t \pm|\Delta \mathbf{x}|)^{2}}-\frac{\delta_{E 3} \Delta t}{(\Delta t \pm|\Delta \mathbf{x}|)^{3}}\right\}\right]
\end{aligned}
$$


where $I_{n}\left(u_{0}^{ \pm}\right)=\int_{0}^{u_{0}^{ \pm}} d u u^{n} e^{-i u}$ with $u_{0}^{ \pm}=u_{\mid k_{\max }}$. In order to perform perturbative expansion in (36), we have assumed $l_{\star} k_{\max } \Delta t \ll(\Delta t \pm|\Delta \mathbf{x}|)$. For a given characteristic length scale $(\Delta t \pm|\Delta \mathbf{x}|)$ which is associated with two-point function, we can choose appropriately large $k_{\max }$, such that $u_{0}^{ \pm} \gg 1$ yet $k_{\max } l_{\star} \ll 1$. This in turn restricts the domain of perturbation in which $l_{\star} \Delta t \ll(\Delta t \pm|\Delta \mathbf{x}|)^{2}$. By introducing a small parameter $\epsilon$ again as a regulator, we can evaluate the integral approximately as

$$
I_{n}^{\epsilon}\left(u_{0}^{ \pm}\right) \approx \frac{\Gamma(n+1)}{(i+\epsilon)^{n+1}},
$$

where we have ignored terms which are exponentially small as $u_{0}^{ \pm}$is large but finite. It is now straightforward to express polymer corrected two-point function, including leading order perturbative correction terms as

$$
G^{\text {poly }}\left(x, x^{\prime}\right) \simeq \frac{(1-i \epsilon)^{-1}}{4 \pi^{2} \Delta x^{2}}\left[1+\frac{2 i \delta^{\text {poly }} l_{\star} \Delta t}{(1-i \epsilon) \Delta x^{2}}\right]
$$

where $\delta^{\text {poly }}=2 \delta_{c 3}+\delta_{E 3}\left[1+4\left(\Delta t^{2} / \Delta x^{2}\right)\right]$. We note that in the limit $l_{\star} \rightarrow 0$, polymer corrected two-point function (38) reduces to Fock space two-point function (25). Leading order polymer correction to the two-point function is of $\mathcal{O}\left(l_{\star}\right)$. However, being purely imaginary number, leading order polymer corrections to the modulus of two-point function which may have direct observational relevance, are of $\mathcal{O}\left(l_{\star}^{2}\right)$. We also note that leading order polymer correction terms explicitly violate Lorentz invariance.

\section{RINDLER TWO-POINT FUNCTION}

Let us consider a thermal detector which is moving along Rindler observer and is located at a spatial position $\vec{\xi}_{0}$ in Rindler frame. If one assumes that the detector is at thermal equilibrium with a reservoir of temperature $\mathrm{T}$ then thermal two-point function (44) associated with the detector is

$$
\mathcal{G}\left(\tau, \tau^{\prime}\right) \equiv\left\langle\hat{\phi}\left(\tau, \vec{\xi}_{0}\right) \hat{\phi}\left(\tau^{\prime}, \vec{\xi}_{0}\right)\right\rangle_{\beta}
$$

For such a detector, KMS condition (5) then becomes

$$
\mathcal{G}\left(\tau, \tau^{\prime}\right)=\mathcal{G}\left(\tau^{\prime}, \tau+i \beta\right)
$$

For simplicity we assume that the detector is located at the origin of Rindler frame i.e. $\vec{\xi}_{0}=(0,0,0)$ and we choose $\tau^{\prime}=0$. Using time evolution of the field operator $\hat{\phi}\left(\tau, \vec{\xi}_{0}\right)$, one can show that $\mathcal{G}(\tau, 0)=\mathcal{G}(0,-\tau)$. Therefore by defining $\mathcal{G}(\tau) \equiv \mathcal{G}(0, \tau)$, we can further simplify KMS condition (40) as

$$
\mathcal{G}(-\tau)=\mathcal{G}(\tau+i \beta)
$$

Now using equation (2), trajectory of the detector can be expressed as $x_{d}(\tau)=(\sinh a \tau / a, \cosh a \tau / a, 0,0)$. So
Fock space two-point function (25) along the trajectory of the detector can be written as

$$
G(\tau) \equiv G\left(x_{d}(\tau), x_{d}(0)\right)=\frac{a^{2}(1-i \epsilon)^{-1}}{8 \pi^{2}(1-\cosh a \tau)} .
$$

Clearly, two-point function (42) satisfies the condition $G(-\tau)=G(\tau+i \beta)$ with $\beta=2 \pi / a$. In other words, two-point function computed in Fock vacuum but when viewed from a uniformly accelerating frame i.e. Rindler frame, appears to satisfy KMS condition (41). Therefore, one may conclude that with respect to Rindler observer, Fock vacuum appears like a thermal reservoir of temperature $T=a / 2 \pi k_{B}$ which is precisely equal to Unruh temperature.

Following similar setup as in Fock space, polymer corrected two-point function (38) along the trajectory of the detector in Rindler frame can be expressed, within the domain of perturbation given by $l_{\star} \ll \Delta t(\tau)$ and $a^{2} l_{\star} \Delta t(\tau) \ll 1$, as

$$
G^{\text {poly }}(\tau)=\frac{a^{2}(1-i \epsilon)^{-1}}{8 \pi^{2}(1-\cosh a \tau)}\left[1+\Delta G_{\star}^{(1)}+\mathcal{O}\left(l_{\star}^{2}\right)\right],
$$

where

$$
\Delta G_{\star}^{(1)}=\frac{i l_{\star} a \sinh a \tau\left[2 \delta_{c 3}-\delta_{E 3}(1+2 \cosh a \tau)\right]}{(1-i \epsilon)(1-\cosh a \tau)} .
$$

We note that unlike in the case of Fock space, polymer corrected two-point function (43) does not satisfy KMS condition (41) given

$$
G^{\text {poly }}(-\tau) \neq G^{\text {poly }}(\tau+i \beta) .
$$

Therefore, polymer corrected two-point function along Rindler trajectory looses its thermal interpretation. From equation (43, 44), it is clear that this violation of KMS condition occurs precisely due to the polymer correction terms. In particular, polymer correction term involving $\delta_{E 3}$ makes dominant contribution to the violation of KMS condition as it contains cosh $a \tau$ term. Contribution from the term involving $\delta_{c 3}$ is comparatively negligible. In other words, polymer corrections to energy spectrum of Fourier modes is the primary cause that leads to the violation of KMS condition. This violation also does not depend on any specific numerical values of $\delta_{c 3}$ and $\delta_{E 3}$. KMS condition is restored if one takes the limit $l_{\star} \rightarrow 0$.

We may note here that unlike Fock quantization there are infinitely many non-vanishing coefficients $c_{n}$ in polymer quantization. Further, the energy gaps $\Delta E_{n}$ depend non-linearly on $n$. Therefore, although we have used a particular superselected sector, given the form of equation (17), it is highly unlikely that any other choice of superselection could have preserved KMS condition.

\section{DISCUSSIONS}

In quantum statistical mechanics, Gibbs ensemble average of a two-point function which is in equilibrium with 
a thermal reservoir, satisfies KMS condition. This property is often used in reverse to argue that if a two-point function computed in a given state satisfies KMS condition then the state appears as a thermal state to the concerned observer. As an example, expectation value of number density operator in Fock vacuum state with respect to a uniformly accelerating observer i.e. Rindler observer, turns out to be a Plank distribution with temperature $T=a / 2 \pi k_{B}$. One can arrive at the same conclusion from the property of two-point function associated with Rindler observer. In particular, two-point function along Rindler trajectory satisfies KMS condition with KMS period along imaginary time being $\beta=2 \pi / a$. This in turns implies corresponding reservoir temperature to be $T=a / 2 \pi k_{B}$.

In this paper, by computing leading order perturbative corrections from polymer quantization, we have shown that polymer corrected two-point function along Rindler trajectory violates KMS condition. Consequently, corresponding two-point function looses its thermal interpretation. This violation is caused by polymer correction terms which are not Lorentz invariants. Intuitively, this loss of thermal characteristic can also be understood from the following arguments. A non-periodic function can be viewed as a periodic function but with a infinite period. In this sense, the 'loss of KMS periodicity with a twist' can be viewed as if KMS period $\beta$ is becoming infinity. This in turn implies that corresponding temperature $T(\sim 1 / \beta)$ is becoming zero. Therefore, the result shown here is consistent with the result of [24] where it is shown using method of Bogoliubov transformation that Unruh effect is absent in polymer quantization. In both methods, polymer correction to the energy spectrum of Fourier modes that leads to the loss of thermal characteristic.

In reference [28], the author has claimed that since
LQG would reproduce Fock space two-point function at zeroth order then it should satisfy KMS condition. Therefore, 'LQG predicts Unruh effect' 28]. This argument is flawed as it tries to completely ignore all possible polymer corrections involving the new scale $l_{\star}$. In fact, we have shown here that violation of KMS condition occurs precisely due to polymer correction terms. Reproduction of Fock-space two-point function at zeroth order is a minimum requirement of viability and it does not imply that polymer quantization would predict exactly same physics as in Fock space.

We emphasize that the violation of KMS condition in Rindler frame as shown here, may not necessarily imply that there will be a violation of KMS condition in the context of black hole spacetime. There are several properties and steps which have been used here may not go through in case of a black hole spacetime [29]. Nevertheless, it would be interesting to carry out a similar analysis for a black hole space time. Based on the results shown here, we would like to reiterate that experimental detection of Unruh effect potentially can be used to either verify or rule out certain candidate theory of quantum gravity which affects matter quantization as described here. We conclude by acknowledging that several experimental proposals have been made in literature to test Unruh effect in laboratory [30 32].

\section{Acknowledgments}

We would like to thank Ritesh Singh for discussions. GS would like to thank UGC for supporting this work through a doctoral fellowship.
[1] S. A. Fulling, Phys.Rev. D7, 2850 (1973).

[2] W. Unruh, Phys.Rev. D14, 870 (1976).

[3] L. C. Crispino, A. Higuchi, and G. E. Matsas, Rev.Mod.Phys. 80, 787 (2008), arXiv:0710.5373.

[4] S. De Bièvre and M. Merkli, Classical and Quantum Gravity 23, 6525 (2006).

[5] S. Takagi, Progress of Theoretical Physics Supplement 88, 1 (1986).

[6] P. Longhi and R. Soldati, Phys.Rev. D83, 107701 (2011), arXiv:1101.5976.

[7] N. D. Birrell and P. C. W. Davies, Quantum fields in curved space, 7 (Cambridge university press, 1984).

[8] R. M. Wald, Quantum field theory in curved spacetime and black hole thermodynamics (University of Chicago Press, 1994).

[9] P. Nicolini and M. Rinaldi, Phys.Lett. B695, 303 (2011), arXiv:0910.2860.

[10] T. Padmanabhan, Rept.Prog.Phys. 73, 046901 (2010), arXiv:0911.5004.

[11] I. Agullo, J. Navarro-Salas, G. J. Olmo, and L. Parker, Phys.Rev. D77, 124032 (2008), arXiv:0804.0513.
[12] R. Haag, N. Hugenholtz, and M. Winnink, Commun.Math.Phys. 5, 215 (1967).

[13] N. Hugenholtz (1971).

[14] O. Bratteli and D. W. Robinson, Operator Algebras and Quantum Statistical Mechanics II (Springer, New York, 1981).

[15] S. Fulling and S. Ruijsenaars, Physics Reports 152, 135 (1987).

[16] W. Troost and H. Van Dam, Phys.Lett. B71, 149 (1977).

[17] W. Troost and H. van Dam, Nucl.Phys. B152, 442 (1979).

[18] A. Ashtekar, S. Fairhurst, and J. L. Willis, Class.Quant.Grav. 20, 1031 (2003), gr-qc/0207106.

[19] H. Halvorson, Studies in history and philosophy of modern physics 35, 45 (2004).

[20] A. Ashtekar and J. Lewandowski, Class.Quant.Grav. 21, R53 (2004), gr-qc/0404018.

[21] C. Rovelli, Quantum Gravity, Cambridge Monographs on Mathematical Physics (Cambridge University Press, 2004).

[22] T. Thiemann, Modern Canonical Quantum General Rela- 
tivity, Cambridge Monographs on Mathematical Physics (Cambridge University Press, 2007).

[23] G. M. Hossain, V. Husain, and S. S. Seahra, Phys.Rev. D82, 124032 (2010), arXiv:1007.5500.

[24] G. M. Hossain and G. Sardar (2014), arXiv:1411.1935.

[25] W. Rindler, Am.J.Phys. 34, 1174 (1966).

[26] M. Abramowitz and I. Stegun, Handbook of Mathematical Functions: With Formulas, Graphs, and Mathematical Tables, Applied mathematics series (Dover Publications, 1964).

[27] J. F. Barbero G., J. Prieto, and E. J. Villaseor,
Class.Quant.Grav. 30, 165011 (2013), arXiv:1305.5406. [28] C. Rovelli (2014), arXiv:1412.7827.

[29] D. Campo and N. Obadia (2010), arXiv:1003.0112.

[30] R. Schutzhold, G. Schaller, and D. Habs, Phys.Rev.Lett. 97, 121302 (2006), quant-ph/0604065.

[31] R. Schutzhold, G. Schaller, and D. Habs, Phys.Rev.Lett. 100, 091301 (2008).

[32] M. Aspachs, G. Adesso, and I. Fuentes, Phys.Rev.Lett. 105, 151301 (2010), arXiv:1007.0389. 